\title{
Association Between Receipt of Brief Alcohol Intervention and Quality of Care among Veteran Outpatients with Unhealthy Alcohol Use
}

\author{
Joseph A. Simonetti, MD, MPH 1,2,3,5, Gwen T. Lapham, PhD, MPH, MSW ${ }^{2,4}$, and \\ Emily C. Williams, $P h D, M P H^{2,3}$
}

'Department of Medicine, Division of General Internal Medicine, University of Washington, Seattle, WA, USA; ${ }^{2}$ Seattle-Denver Center of Innovation, VA Puget Sound Healthcare System, VA Health Services Research \& Development, Seattle, WA, USA; ${ }^{3}$ Department of Health Services, School of Public Health, University of Washington, Seattle, WA, USA; ' ${ }^{4}$ Group Health Research Institute, Seattle, WA, USA; ${ }^{5}$ VA Puget Sound Healthcare System, Center of Innovation for Veteran-Centered Value-Driven Care, Seattle, WA, USA.

BACKGROUND: Brief alcohol intervention, including advice to reduce or abstain from drinking, is widely recommended for general medical outpatients with unhealthy alcohol use, but it is challenging to implement. Among other implementation challenges, providers report reluctance to deliver such interventions, citing concerns about negatively affecting their patient relationships.

OBJECTIVE: The purpose of this study was to determine whether patient-reported receipt of brief intervention was associated with patient-reported indicators of highquality care among veteran outpatients with unhealthy alcohol use.

DESIGN: Cross-sectional secondary data analysis was performed using the Veterans Health Administration (VA) Survey of Healthcare Experiences of Patients (SHEP).

PARTICIPANTS: The study included veteran outpatients who (1) responded to the outpatient long-form SHEP (2009-2011), (2) screened positive for unhealthy alcohol use (Alcohol Use Disorders Identification Test-Consumption (AUDIT-C) questionnaire score $\geq 3$ for women, $\geq 4$ for men), and (3) responded to questions assessing receipt of brief intervention and quality of care.

MAIN MEASURES: We used logistic regression models to estimate the adjusted predicted prevalence of reporting two indicators of high-quality care-patient ratings of their VA provider and of overall VA healthcare (range 010 , dichotomized as $\geq 9$ indicating high quality)—for both patients who did and did not report receipt of brief intervention (receiving alcohol-related advice from a provider) within the previous year.

KEY RESULTS: Among 10,612 eligible veterans, $43.8 \%$ reported having received brief intervention, and $84.2 \%$ and $79.1 \%$ rated their quality of care as high from their provider and the VA healthcare system, respectively. In adjusted analyses, compared to veterans who reported receiving no brief intervention, a higher proportion of veterans reporting receipt of brief intervention rated the quality of healthcare from their provider $(86.9 \%$ vs. $82.0 \%$, $p<0.01)$ and the VA overall $(82.7 \%$ vs. $75.9 \%, p<0.01)$ as high.

Received July 25, 2014

Revised January 14, 2015

Accepted January 23, 2015

Published online February 18, 2015
CONCLUSIONS: In this cross-sectional analysis of veterans with unhealthy alcohol use, a higher proportion of those who reported receipt of brief intervention reported receiving high-quality care compared to those who reported having received no such intervention. These findings do not support provider concerns that delivering brief intervention adversely affects patients' perceptions of care.

KEY WORDS: Alcoholism and addictive behavior; Quality assessment; Veterans; Patient satisfaction.

J Gen Intern Med 30(8):1097-104

DOI: $10.1007 / \mathrm{s} 11606-015-3218-5$

(c) Society of General Internal Medicine 2015

\section{BACKGROUND}

Unhealthy alcohol use is common among general medical outpatients, ${ }^{1,2,3}$ and is associated with tremendous societal costs $^{4}$ and significant patient morbidity and mortality. ${ }^{5-8}$ Brief alcohol intervention, including advice to reduce or abstain from drinking, has demonstrated efficacy in reducing alcohol consumption among general medical outpatients with unhealthy alcohol use identified by population-based screening. ${ }^{9}-11$ As such, population-based screening followed by brief intervention for those who screen positive is widely recommended for general outpatient populations. ${ }^{12-14}$

Despite these recommendations, implementation of this strategy has been challenging, ${ }^{15-17}$ and population-based screening followed by brief intervention has not been widely adopted nationally. ${ }^{18}$ Among a number of barriers to delivering brief intervention, ${ }^{19-22}$ providers have cited concerns that patients may prefer not to discuss their drinking or may react negatively to such conversations. ${ }^{19-21,23-25}$ In other words, providers are concerned that these discussions may compromise their relationships with patients. However, a previous study among general outpatients with unhealthy alcohol use found that those who reported receipt of brief intervention rated their care as higher quality than those not reporting brief intervention. ${ }^{26}$ Importantly, that study was conducted among patients who consented to participate in a clinical trial focused on their alcohol use and thus may have been seeking feedback on their drinking. Therefore, it is unknown whether receipt of 
brief intervention is associated with patient-reported indicators of high-quality care among a more general sample of outpatients with unhealthy alcohol use.

The Veterans Health Administration (VA) has implemented population-based screening for unhealthy alcohol consumption using the validated Alcohol Use Disorders Identification Test-Consumption (AUDIT-C) Questionnaire, ${ }^{27}$ and in 2007 announced a national performance measure to incentivize brief intervention for all patients with AUDIT-C scores $\geq$ $5 .{ }^{27}$. Despite the challenges that other systems have had in implementing population-based screening and brief intervention, the majority of active primary care patients in the VA are screened annually for unhealthy alcohol use, and among those for whom brief intervention is incentivized, more than $75 \%$ have brief intervention documented in their electronic medical record. ${ }^{28,29}$ Additionally, the VA has increased its focus on delivering patient-centered care, ${ }^{30}$ and now routinely measures the experiences and perceptions of VA care among a large national sample of veteran outpatients, including the receipt of brief alcohol interventions as part of their care. ${ }^{31,32}$ As such, the VA provides a unique opportunity to assess the relationship between receipt of brief intervention and patient-reported indicators of high-quality care among a general sample of outpatients. The aim of this study was to assess, from among a sample of VA outpatients who responded to a mailed patient satisfaction survey and screened positive for unhealthy alcohol use, whether patient-reported receipt of brief intervention was associated with patient-reported indicators of care quality.

\section{METHODS}

\section{Data Source and Study Sample}

For this cross-sectional analysis, we used secondary quality improvement data collected by the VA Office of Analytics and Business Intelligence. Specifically, we obtained data from the long-form version of the VA Survey of Healthcare Experiences of Patients (SHEP), ${ }^{33}$ an ongoing survey of veterans receiving care at VA facilities. The outpatient SHEP survey is administered using a stratified random sample withoutreplacement design and is fielded monthly to a fixed percentage of veterans who visited an outpatient facility during the previous month. The survey assesses sociodemographic characteristics, health status, and perceptions and experiences of care, including patient-reported indicators of quality of care, using measures from the Consumer Assessment of Healthcare Providers and Systems (CAHPS) Clinician \& Group Survey. ${ }^{34}$ The long-form version of the outpatient survey, which is sent to a $10 \%$ random sample of surveyed patients, includes alcohol screening questions and a question regarding receipt of alcohol-related advice from a provider.

We included unique veteran outpatients who met the following criteria: (1) responded to the outpatient long-form survey between October 2008 and September 2011 (fiscal years 2009-2011), (2) screened positive for unhealthy alcohol use on the 3-item AUDIT-C included in the survey, based on validated cut points (score $\geq 3$ for women, $\geq 4$ for men; Appendix Table; ${ }^{35,36}$ ), and (3) responded to a question regarding receipt of alcohol-related advice from a VA provider within the past year and to two questions assessing patient ratings of both their VA provider and overall VA healthcare. Response rates to the long-form SHEP were $55 \%, 53 \%$, and $51 \%$ in 2009, 2010, and 2011, respectively. The institutional review board of the VA Puget Sound Healthcare System approved this study.

\section{Measures}

Exposure of Interest: Receipt of Brief Intervention. Receipt of brief intervention was defined as a "yes" response to the following question: "In the past 12 months, has a VA doctor or other VA healthcare provider advised you about your drinking (to drink less or not to drink alcohol)?" This question has previously been used as a proxy for brief intervention because alcohol-related advice is a central component of evidencebased brief intervention. ${ }^{10,37}$

Primary Outcomes: Patient-Reported Indicators of HighQuality Care. The following patient-reported indicators of high-quality care comprised the primary study outcomes: 1) patient report of high-quality care from their VA provider and 2) patient report of high-quality care from the VA. We assessed these outcomes using two survey questions from the CAHPS Clinician \& Group Survey: "Using any number from 0 to 10 , where 0 is the worst personal doctor/nurse possible and 10 is the best personal doctor/nurse possible, what number would you use to rate your personal VA doctor/nurse?" and "Using any number from 0 to 10 , where 0 is the worst healthcare possible and 10 is the best healthcare possible, what number would you use to rate all your VA healthcare in the last 12 months?" Potential scores ranged from 0 to 10 , which we dichotomized $\geq 9$ according to CAHPS guidelines as indicative of high quality. ${ }^{38}$

Potential Confounders. Through a literature search, we identified several patient characteristics as a priori potential confounders and included those available from SHEP data as covariates based on their associations with receipt of brief interventions and patient-reported quality-of-care ratings. ${ }^{31,34,37,39-41}$ These included age, gender, race/ethnicity (non-Hispanic white, non-Hispanic black, Hispanic, or other), educational attainment (less than high school, high school, General Educational Development Test [GED], or 2-year degree; 4-year degree; or greater than 4-year degree), and Short Form-1 self-reported health status (poor, fair, good, very good, or excellent). In addition, we included severity of unhealthy alcohol use, as previous studies have demonstrated associations between greater alcohol consumption and receipt of brief interventions, ${ }^{37,39,40}$ and severity may also be associated with patient-reported quality-of-care ratings. Therefore, we categorized scores on the 3 -item AUDIT-C as mild, moderate, 
severe, or very severe unhealthy alcohol use based on previously established AUDIT-C ranges of 3/4-5, 6-7, 8-9, and $10-12$, respectively. ${ }^{42}$

Statistical Analysis. To describe the study sample, we summarized characteristics of the overall sample and used $t$ tests and chi-square tests to compare the characteristics of respondents who reported receiving brief intervention with those who did not.

We then used unadjusted and adjusted analyses to estimate the association between patient-reported receipt of brief intervention and each patient-reported outcome: high-quality care from their VA provider and high-quality care from the VA. In unadjusted analyses, we used chi-square tests to compare the prevalence of the two outcome measures between the two groups of respondents. In adjusted analyses, we used logistic regression with generalized estimating equations and robust error variance to estimate the adjusted predicted prevalence of each outcome measure for patients who did and did not report receiving brief intervention. Estimates were obtained using recycled predictions, which holds all covariates constant and allows brief intervention to vary. ${ }^{43,44}$ Models were adjusted for all covariates and were clustered to account for correlation of outcomes at the facility level.

Although brief intervention is recommended for all patients with unhealthy alcohol use, the VA's performance measure incentivizes brief intervention only for patients with AUDIT-C scores $\geq 5,{ }^{28,45}$ and so we repeated all main analyses in the subgroup of respondents with AUDIT-C scores $\geq 5$. Lastly, we performed two sensitivity analyses to address survey nonresponse. First, we investigated potential bias based on partial response by comparing sample characteristics between the full study sample and SHEP respondents who screened positive for unhealthy alcohol use but did not respond to either outcome question ( $n=342$, Fig. 1 ). Second, we addressed general survey non-response by repeating main analyses weighted to account for non-response. Weights were developed by the VA's Office of Analytics and Business Intelligence to account for sampling design and non-response related to age, gender, and clinical site. ${ }^{31}$ Large weights were truncated at 500 to ensure individuals with outlying weights did not largely drive results $(n=115)$. We used Stata version 12.0 (StataCorp LP, College Station, TX, USA) for all analyses.

\section{RESULTS}

\section{Study Sample and Patient Characteristics}

Of 125,853 veterans who were mailed the long-form SHEP between 2009 and 2011, 66,311 (52.7\%) returned a completed survey and 10,612 (16\%) screened positive for unhealthy alcohol use and met all inclusion criteria (Fig. 1).

The mean age of the study sample was 64 years (SD 12.2); $96 \%$ were men; $81 \%$ were non-Hispanic white; $92 \%$ had at least a high school degree, GED, or 2-year degree; and $70 \%$ rated their health as good, very good, or excellent (Table 1). Sixty-one percent of the sample screened positive for mild unhealthy alcohol use, and $21 \%, 11 \%$, and $8 \%$ screened positive for moderate, severe, and very severe unhealthy alcohol use, respectively. Comparisons of respondent characteristics across receipt of brief intervention are shown in Table 1 .

\section{Receipt of Brief Intervention and Two Indicators of High-Quality Care}

Forty-four percent of the sample reported receipt of brief intervention within the previous 12 months. Eighty-four percent and $79 \%$ of the sample rated the quality of care from their provider and the VA as high, respectively (Table 2). In unadjusted analyses comparing veterans who reported receipt of brief intervention with those who did not, a higher proportion of veterans who received brief intervention rated the quality of care as high from their provider $(85 \%$ vs. $83 \%, p<0.01)$ and the VA overall ( $80 \%$ vs. $78 \%, p<0.01$ ). Similarly, in adjusted analyses, a higher proportion of veterans who reported receipt of brief intervention rated the quality of care as high from both their provider ( $87 \%$ vs. $82 \%, p<0.01)$ and the VA ( $83 \%$ vs. $76 \%, p<0.01)$.

\section{Receipt of Brief Intervention and Two Indicators of High-Quality Care Among Respondents for Whom Brief Intervention is Incentivized in the VA (AUDIT-C Scores $\geq 5$ )}

Among patients with AUDIT-C scores $\geq 5$ (58\% of the sample), $58 \%$ reported receipt of brief intervention, and $83 \%$ and $78 \%$ rated the quality of care from their provider and the VA as high, respectively (Table 2). In unadjusted analyses, a higher proportion of veterans who reported receipt of brief intervention versus those who did not rated the quality of care as high from their provider $(85 \%$ vs. $81 \%, p<0.001)$ and the VA overall ( $80 \%$ vs. 75 $\%, p<0.001)$. In adjusted analyses, a higher proportion of veterans who reported receipt of brief intervention versus those who did not also rated the quality of care as high from both their provider ( $86 \%$ vs. $79 \%, p<0.01)$ and the VA $(81 \%$ vs. $73 \%, p<0.01)$.

\section{Sensitivity Analyses}

Compared to the study sample, SHEP respondents who screened positive for unhealthy alcohol use but did not respond to either outcome question were more likely to be older and male, reported better health status, and screened positive in lower AUDIT-C risk categories $(p<0.05$ all comparisons). In adjusted analyses weighted to account for survey non-response, a higher proportion of veterans who reported receipt of brief intervention versus those who did not also rated the quality of care as high from both their provider $(84 \%$ vs. $78 \%$, $p<0.01)$ and the VA (79 \% vs. $72 \%, p<0.01)$. 


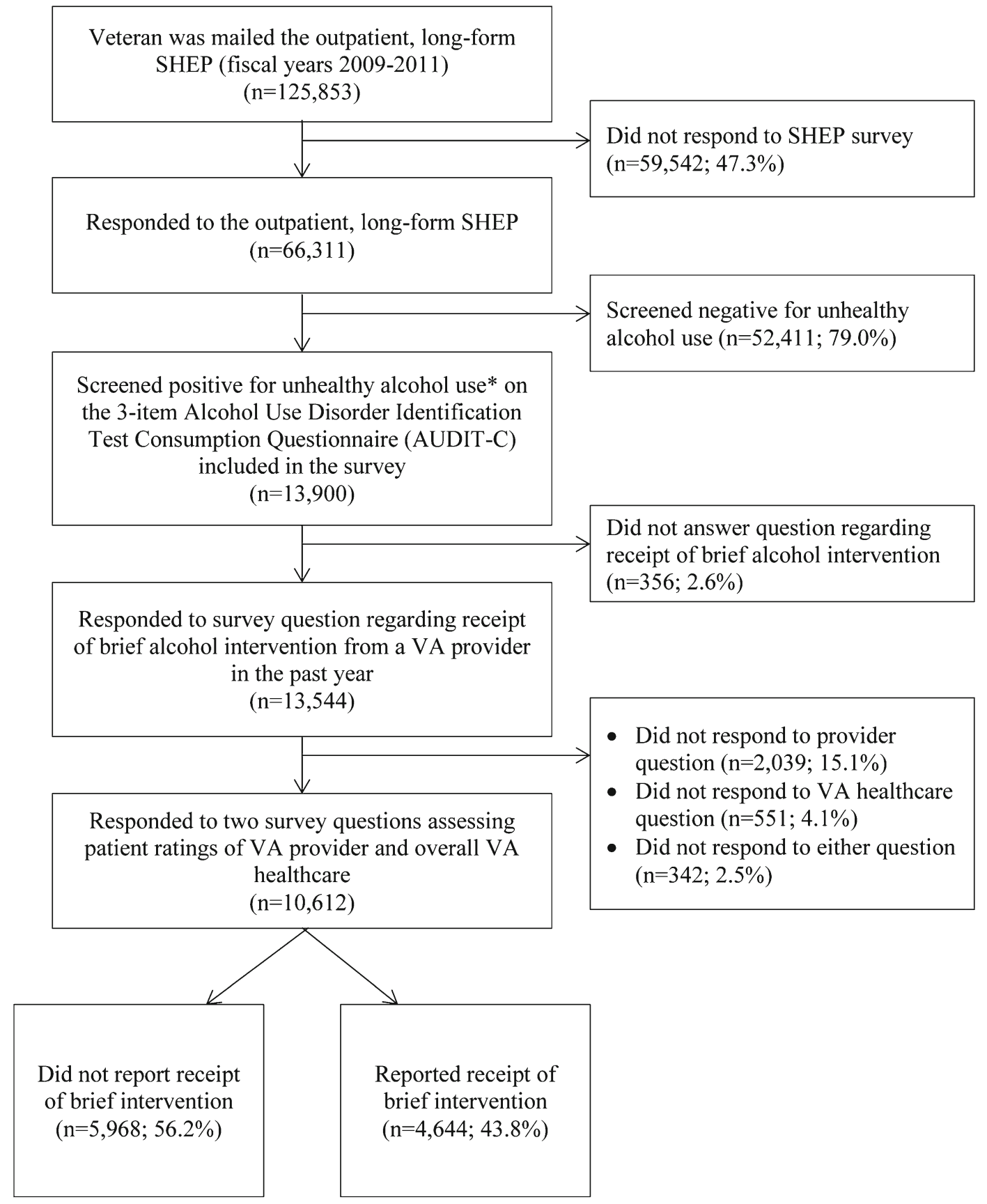

Fig. 1 Selection of the Study Sample. SHEP VA Survey of Healthcare Experiences of Patients * Unhealthy alcohol use defined based on validated AUDIT-C cut points (score $\geq 3$ for women, $\geq 4$ for men)

\section{DISCUSSION}

In this cross-sectional analysis among veterans who screened positive for unhealthy alcohol use on a confidential satisfaction survey, the vast majority rated the quality of care as high from both their provider and the VA in general, and a higher proportion of those who reported receipt of brief intervention within the past year reported receiving high-quality care compared with the group who reported no brief intervention. These findings were robust in analyses limited to veterans for whom brief intervention was incentivized within the VA (AUDIT-C $\geq$
5) as well as in analyses accounting for survey non-response, and are consistent with those of a previous study in a more limited population. ${ }^{26}$ These results do not support provider concerns that delivering such advice adversely affects patient perceptions of their care.

The findings are also consistent with several studies that have assessed patient perceptions of alcohol-related interventions. ${ }^{4-48}$ A previous study of VA outpatients found that more than $80 \%$ of respondents were not "embarrassed, upset, annoyed, or uncomfortable" with questions about their 
Table 1 Sociodemographic, Health Status, and Alcohol Use Characteristics among Veteran Outpatients with Unhealthy Alcohol Use Included in the Study

\begin{tabular}{|c|c|c|c|c|}
\hline \multirow[t]{3}{*}{ Respondent Characteristic } & \multirow{2}{*}{$\begin{array}{l}\text { Reported receipt } \\
\text { of BI } \\
\text { n (\%) }\end{array}$} & \multirow{2}{*}{$\begin{array}{l}\text { Did not report } \\
\text { receipt of BI } \\
\text { n (\%) }\end{array}$} & \multirow{2}{*}{$\begin{array}{l}\text { Total } \\
\text { n (\%) }\end{array}$} & \multirow[t]{3}{*}{$p$ value } \\
\hline & & & & \\
\hline & $4,644(43.8 \%)$ & $5,968(56.2 \%)$ & $10,612(100 \%)$ & \\
\hline Age, mean, years (SD) & $61.3(11.0)$ & $66.5(12.6)$ & $64.2(12.2)$ & $<0.001$ \\
\hline Age category, years, n (\%) & & & & $<0.001$ \\
\hline $18-39$ & $184(4.0)$ & $198(3.3)$ & $382(3.6)$ & \\
\hline $40-59$ & $1,423(30.6)$ & $1,146(19.2)$ & $2,569(24.2)$ & \\
\hline $60-79$ & $2,794(60.2)$ & $3,647(61.1)$ & $6,441(60.7)$ & \\
\hline$\geq 80$ & $243(5.2)$ & $977(16.4)$ & $1,220(11.5)$ & \\
\hline Male gender, n (\%) & 4,512 (97.2) & $5,684(95.2)$ & $10,196(96.1)$ & $<0.001$ \\
\hline Race/ethnicity, n $(\%)^{\mathrm{a}}$ & & & & $<0.001$ \\
\hline Non-Hispanic white & $3,399(75.0)$ & $4,949(85.1)$ & $8,348(80.7)$ & \\
\hline Non-Hispanic black & $546(12.0)$ & $367(6.3)$ & $913(8.8)$ & \\
\hline Hispanic & $355(7.8)$ & $301(5.2)$ & $656(6.3)$ & \\
\hline Other & $235(5.2)$ & $199(3.4)$ & $434(4.2)$ & \\
\hline Educational attainment, $\mathrm{n}(\%)^{\mathrm{a}}$ & & & & $<0.001$ \\
\hline$<$ High school & $412(9.1)$ & $436(7.5)$ & $848(8.2)$ & \\
\hline High school, GED or 2-year degree & $3,474(76.4)$ & $3,895(66.7)$ & $7,369(71.0)$ & \\
\hline Four-year degree & $352(7.7)$ & $724(12.4)$ & $1,076(10.4)$ & \\
\hline$>$ Four-year degree & $308(6.8)$ & $782(13.4)$ & $1,090(10.5)$ & \\
\hline Health status, $\mathrm{n}(\%)^{\mathrm{a}}$ & & & & $<0.001$ \\
\hline Poor or fair & $1,675(36.3)$ & $1,490(25.1)$ & $3,165(30.0)$ & \\
\hline Good, very good, or excellent & $2,944(63.7)$ & 4,433 (74.9) & $7,377(70.0)$ & \\
\hline AUDIT-C risk groups, n (\%) & & & & $<0.001$ \\
\hline Mild unhealthy alcohol use & $1,936(41.7)$ & $4,479(75.1)$ & $6,415(60.5)$ & \\
\hline Score 3-4 (women), 4 (men) & $1,102(23.7)$ & $3,373(56.5)$ & $4,475(42.2)$ & \\
\hline Score $5^{\mathrm{b}}$ & $834(17.9)$ & $1,106(18.5)$ & $1,940(18.3)$ & \\
\hline Moderate unhealthy alcohol use (score 6-7) & $1,270(27.4)$ & $970(16.3)$ & $2,240(21.1)$ & \\
\hline Severe unhealthy alcohol use (score 8-9) & $809(17.4)$ & $348(5.8)$ & $1,157(10.9)$ & \\
\hline Very severe unhealthy alcohol use (score $10-12$ ) & $629(13.6)$ & $171(2.9)$ & $800(7.6)$ & \\
\hline
\end{tabular}

BI brief intervention, $n$ number, SD standard deviation, AUDIT-C Alcohol Use Disorders Identification Test-Consumption Questionnaire, GED General Educational Development Test

Screened positive for unhealthy alcohol use on the 3-item AUDIT-C based on validated cut points (score $\geq 3$ for women, $\geq 4$ for men) Column totals may not sum to $100 \%$ due to rounding.

${ }^{a}$ Due to missing survey item responses, the column totals are lower for racelethnicity $(n=261)$, education $(n=229)$, and health status $(n=70)$.

${ }^{b}$ The VA's performance measure incentivizes brief intervention only for patients who screen positive with AUDIT-C scores $\geq 5$, and an electronic clinical reminder prompts providers to offer brief intervention to all patients with AUDIT-C scores $\geq 5$.

alcohol use. ${ }^{48}$ Another study among a small sample comprising mostly black women receiving primary care in the southern U.S. found that patients had supportive opinions regarding alcohol screening in clinical settings. ${ }^{49}$ In a study among Finnish general outpatients, $81 \%$ agreed that providerdelivered alcohol-related discussions were helpful, ${ }^{46}$ and another study among Australian general outpatients found that highly satisfied patients were more likely to report a previous alcohol-related discussion with their provider. ${ }^{47}$

Population-based screening for unhealthy alcohol use, followed by brief intervention for those who screen positive, is widely recommended for general medical outpatients. ${ }^{12-14}$ These measures, which were established as a standard preventive service by the Affordable Care Act ${ }^{50}$ are incentivized through the VA and other systems, ${ }^{17,27,28}$ and screening is reimbursed by the Centers for Medicare \& Medicaid Services. ${ }^{51}$ Despite this, implementation of these strategies remains a challenge ${ }^{14-17}$ and delivery varies considerably across settings. ${ }^{18}$ These findings may help address system- and provider-level barriers to implementation. Reassuring providers that such conversations do not appear to pose a risk to their patient relationships may encourage delivery of these interventions in clinical settings. In addition, patient-reported indicators of care quality are rapidly being adopted for use in comparative performance metrics and reimbursement decisions in a wide variety of settings. ${ }^{52-54}$ Findings from the study suggest that healthcare delivery systems should not be dissuaded from implementing evidence-based strategies to address unhealthy alcohol use out of concern for harming reimbursement or quality ratings. Given the recent national emphasis on improving the patient-centeredness of care, the large amounts of time and capital being invested in improving patients' experiences in care settings, ${ }^{53,54}$ and a lack of knowledge regarding strategies to improve the care experience, these findings may have relevance for policymakers.

This study demonstrates that in comparisons between patients reporting receipt of brief intervention versus those who did not, a larger percentage of VA patients who received brief intervention also reported receiving high-quality care. However, because of its cross-sectional design, the study cannot clarify whether brief intervention actually improves patients' perceptions of their care. In addition, providers who 
Table 2 Unadjusted and Adjusted Prevalence of Reporting High-Quality Care among Veteran Outpatients with Unhealthy Alcohol Use Who Did and Did Not Receive a Brief Intervention (BI)

\begin{tabular}{|c|c|c|c|c|}
\hline & \multirow{2}{*}{$\begin{array}{l}\text { Received BI } \\
(\mathrm{n}=4,644) \\
\%\end{array}$} & \multirow{2}{*}{$\begin{array}{l}\text { Did not Receive } \\
\text { BI }(\mathrm{n}=\mathbf{5 , 9 6 8 )} \\
\%\end{array}$} & Total $(n=10,612)$ & \multirow[t]{2}{*}{$p$ value } \\
\hline & & & $\%$ & \\
\hline \multicolumn{5}{|c|}{ Unadjusted } \\
\hline \multicolumn{5}{|c|}{ Total Sample: Patients with unhealthy alcohol use* } \\
\hline Rated provider as high quality & 85.3 & 83.4 & 84.2 & $<0.01$ \\
\hline Rated VA healthcare as high quality & 80.2 & 78.2 & 79.1 & $<0.01$ \\
\hline \multicolumn{5}{|c|}{ Subgroup: Patients for whom VA incentivizes $\mathrm{BI} \dagger$} \\
\hline Rated provider as high quality & 84.9 & 80.6 & 83.1 & $<0.001$ \\
\hline Rated VA healthcare as high quality & 79.8 & 75.2 & 77.8 & $<0.001$ \\
\hline & $\%(95 \% \mathrm{CI})$ & $\%(95 \% \mathrm{CI})$ & & $\mathrm{p}$ value \\
\hline \multirow{2}{*}{\multicolumn{5}{|c|}{$\begin{array}{l}\text { Adjusted } \$ \\
\text { Total sample: Patients with unhealthy alcohol use* }\end{array}$}} \\
\hline & & & & \\
\hline Rated provider as high quality & $86.9(85.8-88.0)$ & $82.0(80.8-83.2)$ & - & $<0.01$ \\
\hline Rated VA healthcare as high quality & $82.7(81.5-83.9)$ & $75.9(74.4-77.4)$ & - & $<0.01$ \\
\hline \multicolumn{5}{|c|}{ Subgroup: Patients for whom VA incentivizes $\mathrm{BI} \dagger$} \\
\hline Rated provider as high quality & $85.8(84.6-87.0)$ & $79.3(77.8-80.8)$ & - & $<0.01$ \\
\hline Rated VA healthcare as high quality & $81.3(79.9-82.7)$ & $73.0(71.1-75.0)$ & - & $<0.01$ \\
\hline
\end{tabular}

* Screened positive for unhealthy alcohol use on the 3-item Alcohol Use Disorders Identification Test-Consumption Questionnaire (AUDIT-C) based on validated cut points (score $\geq 3$ for women, $\geq 4$ for men)

$\dagger$ Restricted to respondents with AUDIT-C scores $\geq 5$. The VA's performance measure incentivizes brief intervention only for patients who screen positive with AUDIT-C scores $\geq 5$, and an electronic clinical reminder prompts providers to offer brief intervention to all patients with AUDIT-C scores $\geq 5$.

$\neq$ Prevalence adjusted for age, gender, race, education, self-reported health status, and AUDIT-C risk group

offer comprehensive patient-centered care that addresses multiple dimensions of health may be those who both offer brief alcohol intervention and who receive high ratings from their patients. Thus, high-quality ratings may be a reflection of all dimensions of care rather than a reflection of satisfaction with receiving brief intervention. Further, this study does not address whether perceptions of care influence drinking outcomes after brief intervention. A recent early evaluation of implementation of brief intervention in the VA found that providerdocumented delivery of brief intervention was not associated with resolution of unhealthy alcohol use among outpatients who had repeat alcohol screening. ${ }^{45}$ Perhaps the effectiveness of brief intervention varies based on patient perceptions of their quality of care, and additional research is needed to explore this further.

In this study, $58 \%$ of respondents with AUDIT-C scores $\geq 5$ reported having received brief intervention within the prior year. Other studies using data from VA electronic medical records have suggested that a higher proportion $(76 \%)$ of the VA population receives brief intervention. ${ }^{28,29}$ This discrepancy may be due to a difference between sensitivities of clinical alcohol screening recorded in the medical record and those of patient-administered screening on anonymous surveys. For example, a previous study demonstrated that over 60 $\%$ of VA patients who screened positive for unhealthy alcohol use on SHEP were screened as negative in the clinical setting. ${ }^{55}$ As such, SHEP may be assessing brief intervention among some unhealthy alcohol users who would not have been identified as such clinically, which would lead to a lower estimate of brief intervention using survey data. Nevertheless, we expect the population of patients who screened positive clinically and were eligible for brief intervention to be represented in this sample of SHEP respondents with unhealthy alcohol use.

There were several limitations in this study. First, the findings may be residually confounded by unmeasured factors, including other characteristics of the patient-provider relationship (e.g., duration of relationship), patient-centeredness of the provider, and the overall quality of care provided, including other preventive care services. Second, these findings may not be generalizable to all veterans with unhealthy alcohol use, given the potential for response bias. However, in analyses weighted to account for non-response, findings were unchanged, suggesting that non-response is unlikely to have substantially biased these results. Generalizability to non-VA populations may also be limited, considering that this study included mostly older, male, non-Hispanic white veterans who generally rated the overall quality of their care as high. Third, this study is vulnerable to misclassification bias if respondents incorrectly reported or failed to report receipt of brief intervention. Misclassification may have also resulted from a halo effect, whereby respondents with favorable perceptions of their care may have been more likely to recall or report brief intervention. Fourth, these results may be affected by underreported alcohol use due to social desirability bias. Lastly, we used receipt of alcohol-related advice as a proxy for brief intervention. Although it is a consistent element of efficacious brief intervention, alcohol-related advice is only one component, and we were unable to assess associations between patient perceptions and other components or mechanisms of delivery of brief intervention, such as motivational interviewing.

Notwithstanding these limitations, this is the first study to address whether receipt of brief alcohol intervention is 
associated with patient-reported quality-of-care ratings among a large sample of primary care outpatients with unhealthy alcohol use. Among the veteran outpatients who responded to a mailed survey, high-quality ratings for both their provider and the VA healthcare system were more common among those who reported having received brief intervention from a provider within the previous year than among those who reported no brief intervention. These findings may help to allay provider concerns, and could be incorporated into implementation strategies to improve the delivery of brief intervention in clinical settings.

Acknowledgements: During the preparation of this manuscript, Dr. Simonetti received fellowship support from the Ruth L. Kirschstein National Research Service Award (T32HP10002), administered by the Health Resources and Services Administration. Dr. Williams is supported by a Career Development Award from VA Health Services Research \& Development (CDA 12-276) and is an investigator with the Implementation Research Institute (IRI) at the George Warren Brown School of Social Work at Washington University in St. Louis, MO. IRI is supported through an award from the National Institute of Mental Health (R25 MH080916-01A2) and the Department of Veterans Affairs, Health Services Research \& Development Service, Quality Enhancement Research Initiative (QUERI). The content of this manuscript is solely the responsibility of the authors and does not necessarily represent the official views of any organization. Preliminary findings of this study were presented at the 2014 annual meeting of the Society of General Internal Medicine (San Diego, CA).

Conflicts of Interest: The authors each declare that they have no conflicts of interest to disclose.

Corresponding Author: Joseph A. Simonetti, MD, MPH; VA Puget Sound Healthcare System, VA Health Services Research Development, 1100 Olive Way, Suite 1400, Seattle, WA 98101, USA (e-mail: simonja@uw.edu).

\section{REFERENCES}

1. Williams EC, McFarland LV, Nelson KM. Alcohol consumption among urban, suburban, and rural Veterans Affairs outpatients. J Rural Health. 2012;28(2):202-210.

2. Mertens JR, Weisner C, Ray GT, Fireman B, Walsh K. Hazardous drinkers and drug users in HMO primary care: prevalence, medical conditions, and costs. Alcohol Clin Exp Res. 2005;29(6):989-998.

3. Saitz R. Unhealthy alcohol use. N Engl J Med. 2005;352(6):596-607.

4. Bouchery EE, Harwood HJ, Sacks JJ, Simon CJ, Brewer RD. Bouchery EE, Harwood HJ, Sacks JJ, Simon CJ, Brewer RD. Economic costs of excessive alcohol consumption in the U.S., 2006. Am J Prev Med. 2011;41(5):516-524

5. Centers for Disease Control and Prevention. Alcohol-attributable deaths and years of potential life lost-United States, 2001. MMWR 2004;53(37):866-870. Available at: http://www.cdc.gov/mmwr/preview/ mmwrhtml/mm5337a2.htm. Accessed May 13, 214.

6. Kinder LS, Bryson CL, Sun H, Williams EC, Bradley KA. Alcohol screening scores and all-cause mortality in male Veterans Affairs patients. J Stud Alcohol Drugs. 2009;70(2):253-260.

7. Williams EC, Peytremann-Bridevaux I, Fan VS, et al. The association between alcohol screening scores and health status in male veterans. J Addict Med. 2010;4(1):27-37.

8. Williams EC, Bryson CL, Sun $\mathbf{H}$, et al. Association between alcohol screening results and hospitalizations for trauma in Veterans Affairs outpatients. Am J. Drug Alcohol Abuse. 2012;38(1):73-80.

9. Kaner EF, Beyer F, Dickinson HO, et al. Effectiveness of brief alcohol interventions in primary care populations. Cochrane Database Syst Rev. 2007;2, CD004148.

10. Whitlock EP, Polen MR, Green CA, Orleans T, Klein J. Behavioral counseling interventions in primary care to reduce risky/harmful alcohol use by adults: a summary of the evidence for the U.S. Preventive services task force. Ann Intern Med. 2004;140(7):557-568.

11. Wilk AI, Jensen NM, Havighurst TC. Meta-analysis of randomized control trials addressing brief interventions in heavy alcohol drinkers. J Gen Intern Med. 1997;12(5):274-283

12. U.S. Department of Health and Human Services. Helping patients who drink too much: a clinician's guide, updated 2005 edition. Available at: http://www.niaaa.nih.gov/guide. Accessed June 4, 2014.

13. U.S. Preventive Services Task Force. Screening and behavioral counseling interventions in primary care to reduce alcohol misuse: recommendation statement. Ann Intern Med. 2004;140(7):554-556.

14. Moyer VA; U.S. Preventive Services Task Force. Screening and behavioral counseling interventions in primary care to reduce alcohol misuse: U.S. Preventive Services Task Force recommendation statement. Ann Intern Med. 2013;159(3):210-218.

15. Nilsen $\mathbf{P}$, Aalto $\mathbf{M}$, Bendtsen $\mathbf{P}$, Seppa $\mathbf{K}$. Effectiveness of strategies to implement brief alcohol intervention in primary healthcare. A systematic review. Scand J Prim Health Care. 2006;24(1):5-15.

16. Williams EC, Johnson ML, Lapham GT, et al. Strategies to implement alcohol screening and brief intervention in primary care settings: a structured literature review. Psychol Addict Behav. 2011;25(2):206-214.

17. Substance Abuse and Mental Health Services Administration. Systemslevel implementation of screening, brief intervention, and referral to treatment. Technical Assistance Publication (TAP) Series 33. HHS Publication No. (SMA) 13-4741. Rockville, MD: 2013.

18. Centers for Disease Control and Prevention. Vital signs: Communication between health professionals and their patients about alcohol use-44 states and the District of Columbia, 2011. MMWR 2014;63(01):16-22. Available at: http://www.cdc.gov/mmwr/preview/mmwrhtml/ mm6301a4.htm?s_cid=mm6301a4_w. Accessed May 5, 2014.

19. Beich A, Gannik D, Malterud K. Screening and brief intervention for excessive alcohol use: qualitative interview study of the experiences of general practitioners. BMJ. Oct 19 2002;325(7369):870.

20. Friedmann PD, McCullough D, Chin MH, Saitz R. Screening and intervention for alcohol problems. A national survey of primary care physicians and psychiatrists. J Gen Intern Med. 2000;15(2):84-91

21. Kääriäinen J, Sillanaukee $\mathbf{P}$, Poutanen P, Seppä K. Opinions on alcoholrelated issues among professionals in primary, occupational, and specialized health care. Alcohol Alcohol. 2001;36(2):141-146.

22. Aalto M, Pekuri P, Seppä K. Primary health care nurses' and physicians' attitudes, knowledge and beliefs regarding brief intervention for heavy drinkers. Addiction. 2001;96(2):305-311.

23. Lock CA, Kaner E, Lamont S, Bond S. A qualitative study of nurses' attitudes and practices regarding brief alcohol intervention in primary health care. J Adv Nurs. 2002;39(4):333-342.

24. Cornuz J, Ghali WA, Di Carlantonio D, Pecoud A, Paccaud F. Physicians attitudes towards prevention: importance of intervention-specific barriers and physicians' health habits. Fam Pract 2000;17(6):535-540.

25. Kaner EFS, Heather N, McAvoy BR, Lock CA, Gilvarry E. Intervention for excessive alcohol consumption in primary health care: attitudes and practices of English general practitioners. Alcohol Alcohol. 1999;34(4):559566.

26. Saitz R, Horton NJ, Cheng DM, Samet JH. Alcohol counseling reflects higher quality of primary care. J Gen Intern Med. 2008;23(9): 1482-1486.

27. Bradley KA, Williams EC, Achtmeyer CE, Volpp B, Collins BJ, Kivlahan DR. Implementation of evidence-based alcohol screening in the Veterans Health Administration. Am J Manag Care. 2006; 12(10):597-606.

28. Lapham GT, Achtmeyer CE, Williams EC, Hawkins EJ, Kivlahan DR, Bradley KA. Increased documented brief alcohol interventions with a performance measure and electronic decision support. Med Care. 2012;50(2):179-187.

29. Bradley KA, Johnson ML, Williams EC. Commentary on Nilsen et al.: the importance of asking patients - the potential value of patient report of brief interventions. Addiction. 2011;106(10):1757-1759.

30. Kansagara D, Tuepker A, Joos S, Nicolaidis C, Skaperdas E, Hickam D. Getting performance metrics right: a qualitative study of staff experiences implementing and measuring practice transformation. J Gen Intern Med. 2014/02/21 2014:1-7

31. Wright SM, Craig T, Campbell S, Schaefer J, Humble C. Patient satisfaction of female and male users of Veterans Health Administration services. J Gen Intern Med. 2006;21(Suppl 3):S26-32.

32. Perlin JB, Kolodner RM, Roswell RH. The Veterans Health Administration: quality, value, accountability, and information as transforming strategies for patient-centered care. Am J Manag Care. 2004;10(11 Pt 2):828-836 
33. Survey of Healthcare Experiences of Patients (SHEP). Washington, DC: Office of Quality and Performance, Veterans Health Administration; 2004.

34. CAHPS Overview Clinician \& Group Surveys: Practical options for implementation and use. March 2012. Agency for Healthcare Research and Quality, Rockville, MD. Available at: http://www.ahrq.gov/news/ events/conference/2011/crofton/index.html. Accessed February 21, 2014

35. Bush K, Kivlahan DR, McDonell MB, Fihn SD, Bradley KA. The AUDIT alcohol consumption questions (AUDIT-C): an effective brief screening test for problem drinking. Ambulatory Care Quality Improvement Project (ACQUIP). Alcohol Use Disorders Identification Test. Arch Intern Med 1998;158(16): 1789-1795.

36. Bradley KA, Bush KR, Epler AJ, et al. Two brief alcohol-screening tests from the Alcohol Use Disorders Identification Test (AUDIT): validation in a female Veterans Affairs patient population. Arch. Intern. Med. 2003;163(7):821-829.

37. Burman ML, Kivlahan D, Buchbinder M, et al. Alcohol-related advice for Veterans Affairs primary care patients: Who gets it? Who gives it? J. Stud. Alcohol. 2004;65(5):621-630.

38. Aligning Forces for Quality. How to report results of the CAHPS Clinician \& Group Survey. Available at: http://www.facs.org/ahp/cahps/how-to-report-results.pdf. Accessed December 13, 2013.

39. Kaner EF, Heather N, Brodie J, Lock CA, McAvoy BR. Patient and practitioner characteristics predict brief alcohol intervention in primary care. Br J Gen Pract. 2001;51(471):822-827.

40. Arndt S, Schultz SK, Turvey C, Petersen A. Screening for alcoholism in the primary care setting: are we talking to the right people? J Fam Pract. 2002;51(1):41-46.

41. O'Malley AJ, Zaslavsky AM, Elliott MN, Zaborski L, Cleary PD. Casemix adjustment of the CAHPS hospital survey. Health Serv Res. 2005;40(6 Pt 2):2162-2181.

42. Rubinsky AD, Kivlahan DR, Volk RJ, Maynard C, Bradley KA. Estimating risk of alcohol dependence using alcohol screening scores. Drug Alcohol Depend. 2010;108(1-2):29-36.

43. Basu A, Rathouz PJ. Estimating marginal and incremental effects on health outcomes using flexible link and variance function models. Biostat. 2005;6(1):93-109.

44. Glick, et al. Economic Evaluation in Clinical Trials. Oxford: Oxford University Press; 2007.

45. Williams EC, Chavez LJ, Lapham GT, Rittmueller SE, Achtmeyer CE, Bradley KA. An early evaluation of implementation of brief intervention for unhealthy alcohol use in the U.S. Veterans Health Administration. Addiction. 2014 Apr 29 [epub ahead of print].

46. Aalto M, Seppa K. Usefulness, length and content of alcohol-related discussions in primary health care: the exit poll survey. Alcohol Alcohol. 2004;39(6):532-535.

47. Steven ID, Thomas SA, Eckerman E, Browning C, Dickens E. The provision of preventive care by general practitioners measured by patient completed questionnaires. J Qual Clin Pract. 1999;19(4):195201.

48. Zimmerman M, Farber NJ, Hartung J, Lush DT, Kuzma MA. Screening for psychiatric disorders in medical patients: a feasibility and patient acceptance study. Med Care. 1994;32(6):603-608.

49. Miller PM, Thomas SE, Mallin R. Patient attitudes towards self-report and biomarker alcohol screening by primary care physicians. Alcohol Alcohol. 2006;41(3):306-310.

50. Department of Health and Human Services. Preventive services covered under the Affordable Care Act. Available at: http://www.hhs.gov/ healthcare/facts/factsheets/2010/07/preventive-services-list.html. Accessed June 4, 2014.

51. Center for Medicare \& Medicaid Services (2011). Decision memo for screening and behavioral counseling interventions in primary care to reduce alcohol misuse (CAG-00427N). Available at: http://www.cms.gov/ medicare-coverage-database/details/nca-decision-memo.aspx? NCAId=249 - _ftn29. Accessed March 24, 2014.

52. Center for Medicare \& Medicaid Services. HCAHPS: Patients' Perspectives of Care Survey. Available at: http://www.cms.gov/Medicare/Quality-Initiatives-Patient-Assessment-Instruments/HospitalQualityInits/HospitalHCAHPS.html. Accessed July 24, 2014.

53. Adamy J (12 Oct 2012). U.S. Ties Hospital Payments to Making Patients Happy. The Wall Street Journal. Available at: http://online.wsj.com/ articles/SB10000872396390443890304578010264156073132. Accessed January 12, 2015.

54. Rau J (28 Apr 2011). Medicare to Begin Basing Hospital Payments on Patient-Satisfaction Scores. Kaiser Health News. Available at: http:// kaiserhealthnews.org/news/medicare-hospital-patient-satisfaction. Accessed January 12, 2015.

55. Bradley KA, Lapham GT, Hawkins EJ, et al. Quality concerns with routine alcohol screening in VA clinical settings. J Gen Intern Med. 2011;26(3):299-306.

56. Seale JP, Boltri JM, Shellenberger S, et al. Primary care validation of a single screening question for drinkers. J Stud Alcohol. 2006;67(5):778784

\section{APPENDIX}

Alcohol Use Disorders Identification Test-Consumption Questionnaire

\begin{tabular}{llc}
\hline Question & Answer & Score \\
\hline How often do you have a drink & Never & 0 \\
containing alcohol? & Monthly or less & 1 \\
& $2-4$ times a month & 2 \\
& $2-3$ times a week & 3 \\
& 4 or more times a week & 4 \\
How many standard drinks* & \multicolumn{1}{c}{1 or 2} & 0 \\
containing alcohol do you & 3 or 4 & 1 \\
have on a typical day? & 5 or 6 & 2 \\
& 10 or more & 3 \\
How often do you have six or & Never & 0 \\
more drinks on one occasion? & Less than monthly & 1 \\
& Monthly & 2 \\
& Weekly & 3 \\
& Daily or almost daily & 4
\end{tabular}

*A standard drink is approximately 12 to 14 grams of ethanol, which corresponds to 12 ounces of beer, 5 ounces of wine, or 1.5 ounces of 80proof liquor. $^{3}$

**Unhealthy alcohol use defined using validated cut points (score $\geq 3$ for women, $\geq 4$ for men) 40,56 